\title{
The mechanisms of substance P-mediated migration of bone marrow-derived mesenchymal stem cell-like ST2 cells
}

\author{
MARIA JOSE DUBON $^{1}$ and KI-SOOK PARK ${ }^{2,3}$ \\ ${ }^{1}$ Graduate School of Biotechnology, Kyung Hee University, Yong-In, Gyeonggi 446-701; \\ ${ }^{2}$ East-West Medical Research Institute and ${ }^{3}$ College of Medicine, Kyung Hee University, Seoul 02447, Republic of Korea
}

Received July 2, 2015; Accepted February 17, 2016

DOI: $10.3892 / \mathrm{ijmm} .2016 .2496$

\begin{abstract}
Substance P(SP) is known to induce the mobilization of bone marrow-derived mesenchymal stem cells (BM-MSCs) and thus participates in wound repair. However, the cellular and molecular mechanisms responsible for the SP-mediated migration of BM-MSCs were not fully understood. In the present study, we studied the molecular mechanisms that mediate the migration of the BM-derived MSC-like cell line ST2 in response to SP. Using a migration assay and western blot analysis, we noted that SP induced the chemotactic migration of ST2 cells through the intrinsic activation of extracellular signal-regulated kinases (ERKs) and protein kinase B (Akt), the phosphorylated expression levels of which were increased. We noted that Src is involved in the SP-mediated migration of ST2 cells and that focal adhesion kinase (FAK) was activated in the ST2 cells following SP treatment. Membrane ruffling increased in the ST2 cells after SP treatment, as was clearly demonstrated by immunocytochemical analysis. Importantly, using a blocking antibody against N-cadherin (GC-4), we studied cell migration and noted that SP mediated the migration of the ST2 cells through N-cadherin. The present study thus advanced our understanding of the mechanisms through which SP induces BM-MSC migration.
\end{abstract}

\section{Introduction}

Bone marrow-derived mesenchymal stem cells (BM-MSCs) have previously been shown to participate in the regeneration $(1,2)$ and homeostasis of various tissues (3). Diverse factors, including stromal cell-derived factor-1 (SDF-1) (4), transforming growth factor- $\beta$ (TGF- $\beta$ ) $(5,6)$, platelet-derived growth factor (PDGF) (7) and substance P (SP) $(8,9)$, induce

Correspondence to: Professor Ki-Sook Park, East-West Medical Research Institute, Kyung Hee University, 26 Kyungheedae-ro, Dongdaemun-gu, Seoul 02447, Republic of Korea

E-mail: kisookpark@khu.ac.kr

Key words: mesenchymal stem cell, substance P, ST2 cells, migration, $\mathrm{N}$-cadherin the migration of BM-MSCs to regeneration sites in order to accelerate the wound healing process. Furthermore, the migration of BM-MSCs plays an important role in the establishment of the tumor microenvironment $(10,11)$.

Although the migration of BM-MSCs is essential to many important biological processes, the cellular and molecular mechanisms responsible for BM-MSC migration were not fully understood. Several intracellular signaling pathways have been suggested to act as mediators of BM-MSC migration, including protein kinase B (Akt) and the mitogen-activated protein kinase/extracellular signal-regulated kinase 1/2 (MAPK/ERK 1/2) signaling pathway. These pathways are among the most important signaling pathways that control the growth of various types of cells including BM-MSCs $(12,13)$. Moreover, previous research has suggested that they are also important for the migration of BM-MSCs, as it has been shown that the pharmacological regulation of Akt activity affects BM-MSCs migration (14), and SDF-1 promotes the migration of human BM-MSCs through signal transducer and activator of transcription 3 (STAT3) and ERKs (15).

Integrin-based focal adhesions are an essential part of cell-extracellular matrix interactions, which are regulated to enable cell migration (16). The focal adhesion kinase (FAK) forms physical and functional complexes with other proteins inside the focal adhesions in order to control cell migration in response to various extracellular stimuli $(17,18)$. The Src tyrosine kinase also interacts functionally with proteins of these complexes and regulates the turnover of focal adhesions that directly control cell migration (16). The important role which FAK plays has been shown in relation to the PDGF-mediated migration of BM-MSCs (7).

Cell-cell interactions are also important in cell migration (16). The cadherin family mediates these intercellular interactions through adherens junctions (19). Importantly, the requirement of $\mathrm{N}$-cadherin for cell migration has been demonstrated in cancer cells (20), mammary epithelial cells (21) and the neural crest (22).

Previous research has demonstrated that SP, an 11-aminoacid neuropeptide involved in pain perception, enhances the migration of BM-MSCs to participate in tissue regeneration and wound repair, and that SP upregulated the expression of matrix metalloproteinases (MMPs) in BM-MSCs (9). However, the mechanisms of SP-mediated migration of BM-MSCs were unknown. Thus, in the present study we decided to examine the 
cellular and molecular mechanisms that induce the migration of BM-MSCs in response to SP in vitro using the BM-derived MSC-like cell line ST2.

\section{Materials and methods}

Cell culture. The ST2 cell line was purchased from Riken Cell Bank (Tsukuba, Japan). Cells were cultured at $37^{\circ} \mathrm{C}$ in a humidified incubator containing 5\% $\mathrm{CO}_{2}$ in RPMI 1640 supplemented with $10 \%$ heat-inactivated fetal bovine serum (FBS) and $1 \%$ penicillin/streptomycin $(\mathrm{P} / \mathrm{S})$ (all from Invitrogen, Carlsbad, CA, USA). When they reached $80 \%$ confluence, the cells were harvested using $0.25 \%$ trypsin/EDTA (Invitrogen) and sub-cultured at a ratio of 1:3-1:4. The medium was changed every 3-4 days. Cells at passage 5-8 were used for experiments.

SP. SP, which we used to induce the mobilization of BM-MSCs, was purchased from EMD Millipore (San Diego, CA, USA) and was prepared with $5 \%$ acetic acid (Sigma-Aldrich, St. Louis, MO, USA).

Migration assay. Millicell culture plate inserts (EMD Millipore), $8-\mu \mathrm{m}$ pore size, were coated with type I collagen $(0.5 \mu \mathrm{g} / \mathrm{ml}$; Nitta Gelatin NA Inc., Osaka, Japan) and allowed to dry overnight. After washing the inserts three times with phosphate-buffered saline (PBS), $2.5 \times 10^{4}$ ST2 cells were seeded on the upper chamber of each insert suspended in normal growth media. Cells were incubated for 5-6 h and medium was then changed for Dulbecco's modified Eagle's medium (DMEM; GE Healthcare Life Sciences, Buckinghamshire, UK) including $2 \% \mathrm{FBS}$ and $1 \% \mathrm{P} / \mathrm{S}$. After overnight incubation, $300 \mathrm{nM} \mathrm{SP}$ was added to the lower chamber of each well. When required, the cells were pre-treated for $30 \mathrm{~min}$ with the following antagonists: the NK1 antagonist RP $67580(10 \mu \mathrm{M}$; Tocris Bioscience, Bristol, UK); the Src inhibitor PP2 $(1 \mu \mathrm{M})$; the PI3K inhibitor LY294002 (10 $\mu \mathrm{M})$; the MAPK/ERK kinase (MEK) inhibitor PD98059 $(10 \mu \mathrm{M})$ (all from EMD Millipore) mouse IgG (40 $\mu \mathrm{g} / \mathrm{ml}$; Jackson Immuno Research, Pennsylvania, PA, USA) or monoclonal anti-N-cadherin antibody (clone GC-4, $40 \mu \mathrm{g}$ / ml, Cat. no. C3865; Sigma-Aldrich). After $12 \mathrm{~h}$ incubation, the inserts were fixed using $4 \%$ paraformaldehyde in PBS for $10 \mathrm{~min}$ at room temperature and stained with hematoxylin solution (Sigma-Aldrich) for $30 \mathrm{~min}$. The ST2 cells that remained on the upper chamber membrane were removed with cotton swabs. Micrographic images of the lower chamber membrane were obtained using a light microscope (Nikon Eclipse TS100; Nikon, Tokyo, Japan) and the number of cells on each image was counted using Adobe Photoshop CS6 (Adobe Systems, Inc., San Jose, CA, USA).

Western blot analysis. The ST2 cells were seeded on 6-well plates with normal growth medium and then left to attach for $6 \mathrm{~h}$. The cells were serum starved for $16-18 \mathrm{~h}$ with serum-free DMEM (GE Healthcare Life Sciences) and then treated with $300 \mathrm{nM} \mathrm{SP}$ at different time points. When required, the cells were exposed to inhibitors for $30 \mathrm{~min}$. To obtain the cell lysate, the cells were rinsed twice with ice-cold PBS and incubated with $400 \mu \mathrm{l} 2 \mathrm{X}$ SDS buffer [100 mM Tris-Cl (pH 6.8), $4 \%(\mathrm{w} / \mathrm{v}) \mathrm{SDS}, 0.2 \%(\mathrm{w} / \mathrm{v})$ bromophenol blue, $20 \%$ glycerol, $200 \mathrm{mM} \beta$-mercaptoethanol] for $5 \mathrm{~min}$ at room temperature.
The cell lysate was collected and denatured at $92^{\circ} \mathrm{C}$ for $10 \mathrm{~min}$. Protein samples were subjected to $10 \%$ sodium dodecyl sulfate-polyacrylamide gel electrophoresis (SDS-PAGE). Separated proteins were transferred onto nitrocellulose membranes (Whatman, Dassel, Germany). The membranes were subsequently incubated with primary antibodies against phosphorylated (p-)p44/42 MAPK (ERKs) (Thr202/Tyr204; 1:1,000, Cat. no. 4370), p-Akt (Ser473; 1:4,000, Cat. no. 4060S) (both from Cell Signaling Technology, Danvers, MA, USA), p-FAK (Y397; 1:1,000, Cat. no. 4803) (Abcam, Cambridge, UK), or p-p38 MAPK (Thr180/Tyr182; 1:1,000, Cat. no. 4511) (Cell Signaling Technology). Subsequently, target proteins were detected with horseradish peroxidase (HRP)-conjugated secondary antibodies and an enhanced chemiluminiscence reagent (Millipore, Billerica, MA, USA). Band densities were measured using ImageJ software (National Institutes of Health, Bethesda, MD, USA). The membranes were then re-blotted with antibody against $\alpha$-tubulin (1:20,000, Cat. no. T5168; Sigma-Aldrich) when required after stripping.

Immunocytochemical analysis. For immunofluorescence staining, cells were seeded on type 1 collagen $(1.5 \mu \mathrm{g} / \mathrm{ml}$; Nitta Gelatin NA Inc.) coated coverslips and left to attach for $6 \mathrm{~h}$. After $18 \mathrm{~h}$ serum starvation, cells were treated with $300 \mathrm{nM}$ SP for $30 \mathrm{~min}$. The cells were fixed with $4 \%$ paraformaldehyde in PBS for $10 \mathrm{~min}$ on ice. Following washing with $0.1 \%$ Triton X-100 (USB Corp., Cleveland, OH, USA), blocking solution (5\% non-fat milk in PBS with $0.1 \%$ Triton $\mathrm{X}-100$ ) was added for $30 \mathrm{~min}$ at room temperature. The cells were then incubated with Alexa Fluor 546 phalloidin (1:1,000, cat. no. A22283; Invitrogen) for $30 \mathrm{~min}$. After washing three times with $1 \%$ non-fat milk in PBS with $0.1 \%$ Triton $\mathrm{X}-100$, the samples were mounted using ProLong Gold antifade mounting solution with 4',6-diamidino-2-phenylindole (DAPI; Invitrogen) and left to dry overnight before observation. Images were then captured using a Zeiss LSM 700 confocal microscope (Zeiss, Oberkochen, Germany). The percentage of cells presenting with ruffled edges was determined in 10 random images per each experimental group. Three independent experiments were performed for the analysis.

Statistical analysis. Data are presented as the means \pm standard deviation. The unpaired Student's t-test was applied to evaluate differences between two groups. A p-value $<0.05$ was considered to indicate a statistically significant difference. All statistical analyses were performed using GraphPad version 5.01 software (GraphPad Software, San Diego, CA, USA) (http://www.graphpad.com).

\section{Results}

SP enhances the migration of ST2 cells. A previous study showed that SP induces the mobilization of BM-MSCs in vivo (9). However, the mechanisms involved in the SP-mediated migration of BM-MSCs had not previously been elucidated. We showed in our previous study that SP enhances the migration potential of the BM-derived MSC-like cell line ST2 in a wound healing migration assay (23). In order to investigate the mechanisms involved in the SP-mediated migration of BM-MSCs, we examined the effects of SP on the migration 

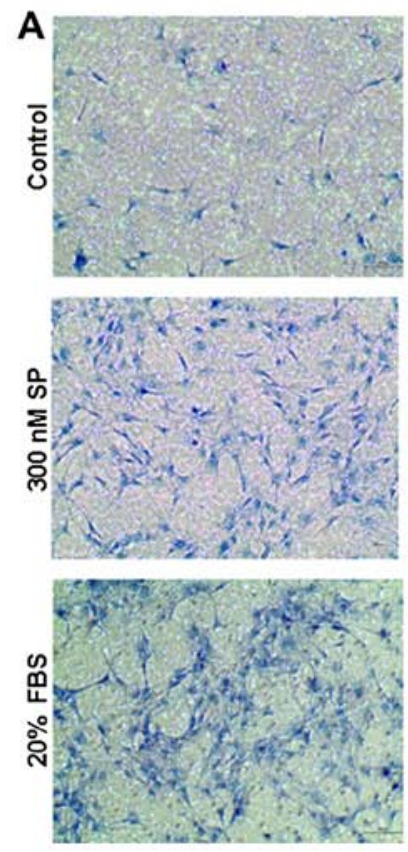
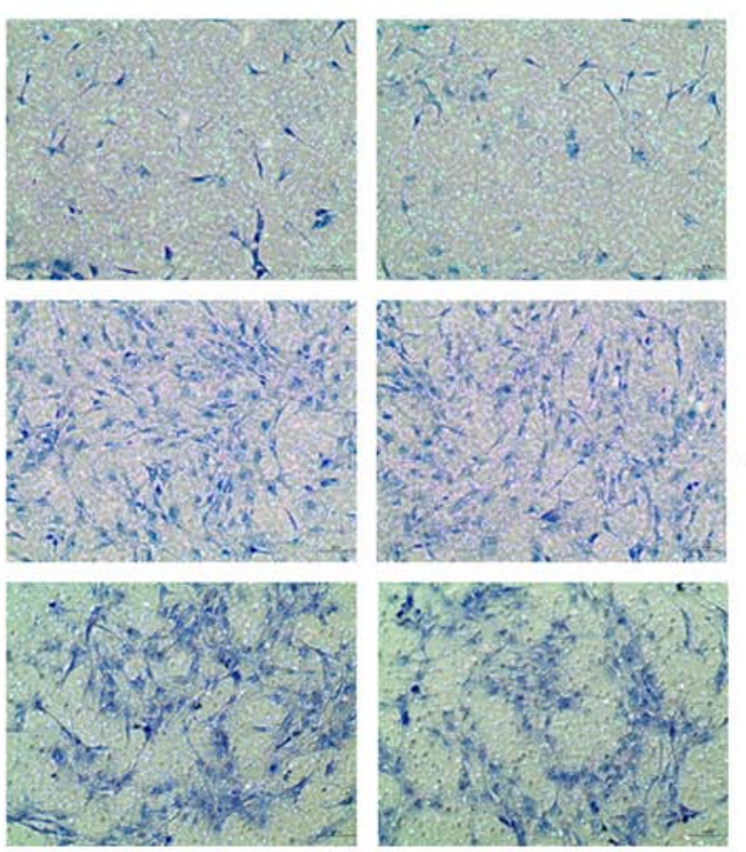
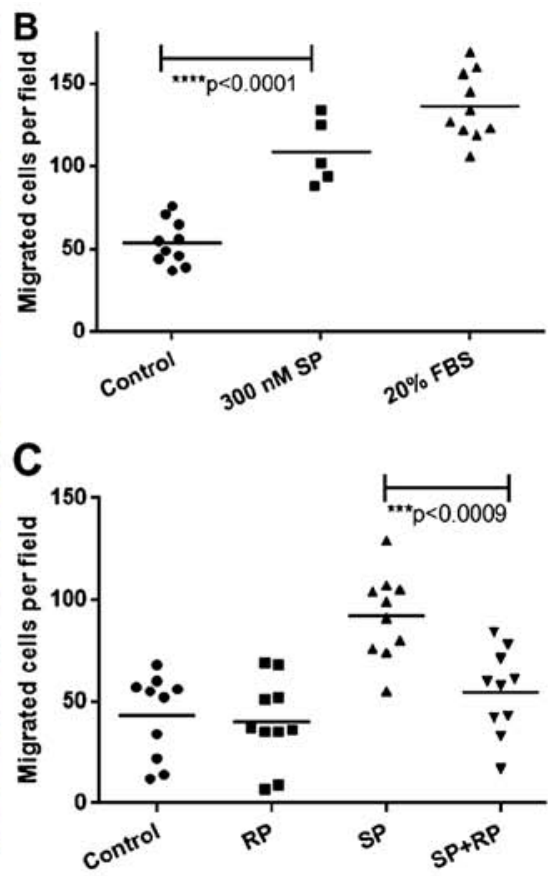

Figure 1. Substance P (SP) induces the migration of ST2 cells. (A) Representative images of migrated ST2 cells after $300 \mathrm{nM}$ SP treatment and (B) the quantitative analysis of the migrated ST2 cells. Growth medium containing 20\% FBS was used as positive control. (C) An SP receptor antagonist inhibited the migration of ST2 cells in response to SP. ST2 cells were pretreated for 30 min with $10 \mu \mathrm{M}$ RP 67580 (RP). Control cells were treated with the appropriate amount of SP solvent (5\%acetic acid). Five fields of the lower chamber membrane were photographed and migrated cells were counted. Scale bar, $100 \mu \mathrm{m}$. Black lines indicate mean values; $\mathrm{p}$-values were calculated using the Student's t-test.

of ST2 cells in vitro using a Millicell migration assay. We observed that SP induced the migration of ST2 cells, as the number of migrated cells was similar to that of the positive control, 20\% FBS (Fig. 1A and B). We confirmed the specific effect of SP on the chemotactic migration of ST2 cells by Millicell migration assay using the antagonist for SP receptor neurokinin-1 (NK-1), RP 67580. Pre-treating ST2 cells with RP 67580 blocked their migration in response to SP (Fig. 1C). These data demonstrate that SP induces the chemotactic migration of ST2 cells. These findings allowed us to use this cell line to further investigate the mechanisms involved.

SP induces the activation of ERKs, Akt, and FAK in ST2 cells. Extracellular signaling through membrane receptors activates several intracellular signaling pathways, which results in changes in cell motility and cell migration. Different pathways have been implicated in the mobilization of BM-MSCs in response to various chemoattractants and growth factors (24). Certain studies have shown that the mitogen-activated protein/ extracellular signal-regulated kinase 1/2 (MAPK/ERK 1/2) signaling pathway is involved in the expression of a wide variety of genes controlling migration and in the migration of MSCs $(25,26)$. We found that SP induced the activation of ERKs (Fig. 2A). It has previously been reported that the phosphoinositide 3-kinase (PI3K)/Akt signaling pathway is involved in both the SDF-1- (27) and the basic fibroblast growth factor (bFGF)-induced migration of MSCs $(28,29)$. In the present study, we observed that SP induced the activation of Akt in ST2 cells, which started $2 \mathrm{~h}$ after SP treatment and persisted up to $9 \mathrm{~h}$ (Fig. 2B). Integrin signaling through FAK has previously been shown to promote cell migration (30). Activation of FAK results in the reorganization of

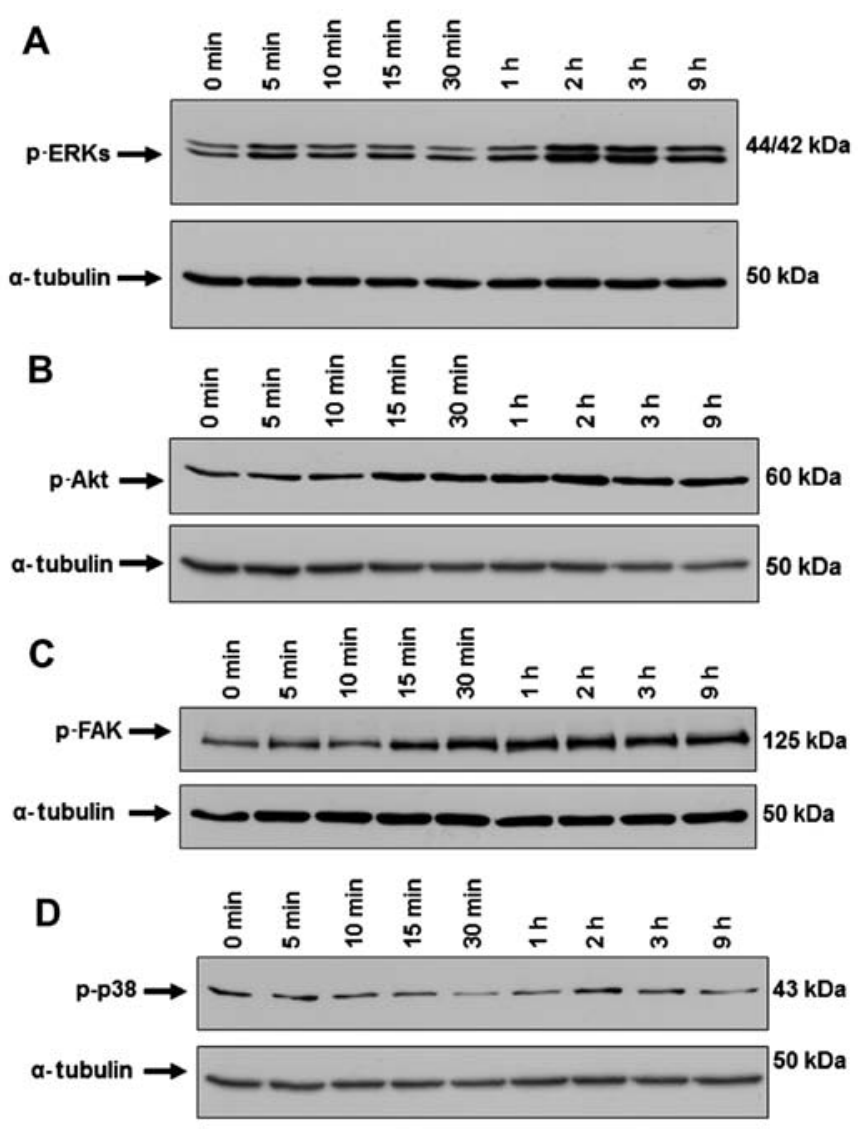

Figure 2. Substance P (SP) increases the levels of phosphorylated extracellular signal-regulated kinases (ERKs), Akt and focal adhesion kinase (FAK). Western blot analysis detected (A) phosphorylated (p-)ERKs, (B) p-Akt, (C) p-FAK or (D) p-p38. ST2 cells were treated with $300 \mathrm{nM}$ SP for the indicated times; $\alpha$-tubulin was used as an internal control. 


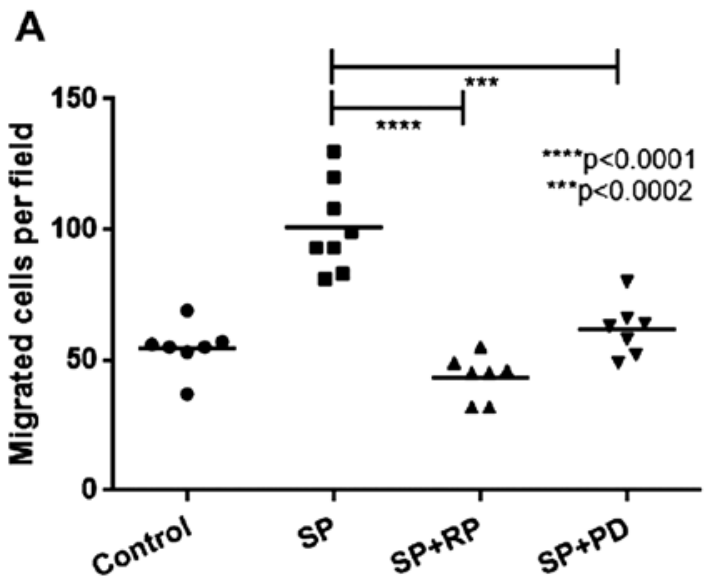

B

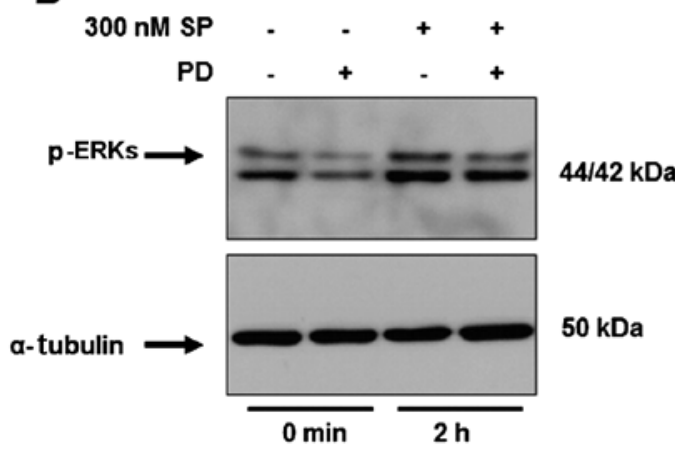

C

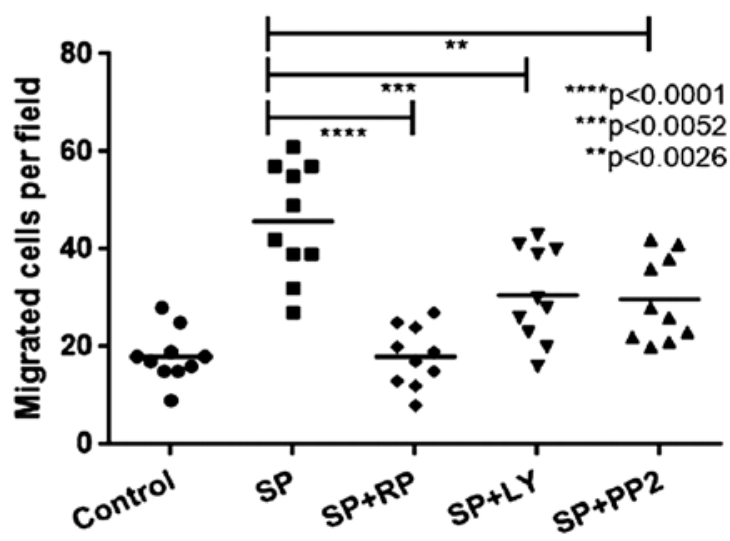

D

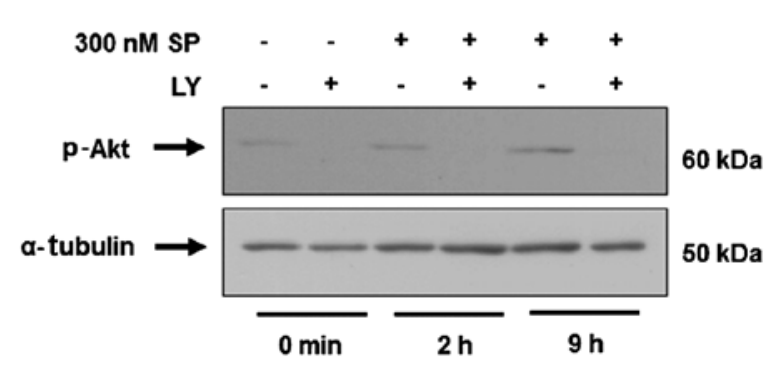

$\mathbf{E}$

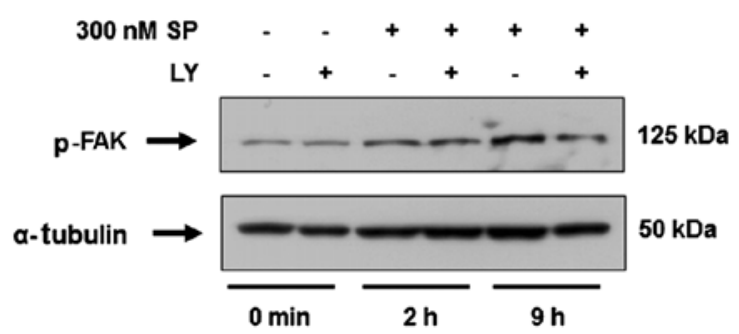

Figure 3. Inhibition of the mitogen-activated protein kinase (MAPK) extracellular signal-regulated kinase (ERK), Akt or Src pathway impairs the migration of ST2 cells induced by substance P (SP). (A and C) The number of migrated ST2 cells in each experimental group. ST2 cells were treated for 30 min with inhibitors prior to treatment with $300 \mathrm{nM} \mathrm{SP}$ and exposure to (A) antagonist for SP receptor neurokinin-1 (RP67580; $10 \mu \mathrm{M}, \mathrm{RP}$ ) or MAPK/ERK kinase (MEK) inhibitor, PD98059 (10 $\mu \mathrm{M}, \mathrm{PD})$, (C) phosphoinositide 3-kinase (PI3K) inhibitor, LY294002 (10 $\mu \mathrm{M}, \mathrm{LY})$, or Src kinase inhibitor, PP2 (1 $\mu \mathrm{M})$. Control cells were treated with the appropriate amount of SP solvent (5\% acetic acid). (B, D and E) Western blot analysis for the levels of (B) p-ERKs, (D) p-Akt or (E) p-FAK following exposure to PD or LY inhibitors and treatment with $300 \mathrm{nM}$ SP. Inhibitors were administered at the concentrations shown for 30 min prior to $300 \mathrm{nM}$ SP treatment for the indicated times. $\alpha$-tubulin was used as an internal control. p-values were calculated using the Student's t-test.

actin filaments and the cytoskeleton, thus inducing MSCs migration (30). The level of p-FAK increased after SP treatment in ST2 cells (Fig. 2C). Studies have suggested that the p38 MAPK pathway participates in the tumor necrosis factor (TNF)- $\alpha$-induced migration of MSCs (31). However, we did not observe any significant change in the level of p-p38 in the ST2 cells after SP treatment (Fig. 2D). Taken together, these data suggest that the intracellular signaling of ERKs, Akt and FAK but not $\mathrm{p} 38$ contributes to the SP-mediated migration of ST2 cells.

Inhibition of ERKs, Akt or Src kinase blocks the migration of $S T 2$ cells in response to SP. To evaluate whether the activation of ERKs in the ST2 cells after SP treatment is required for the migration of ST2 cells in response to SP, we pretreated ST2 cells with the MEK inhibitor, PD98059. Inhibition of p-ERKs by PD98059 significantly reduced the migration of ST2 cells in response to SP, compared with the SP-treated group (Fig. 3A and B). Thus, the activation of the ERK pathway is required for the migration of ST2 cells in response to SP.

We subsequently investigated the role of the Akt pathway in the SP-induced migration of ST2 cells. We noted that the PI3K inhibitor LY294002 significantly decreased the migration of ST2 cells in comparison to SP-treated cells (Fig. 3C) as well as the level of p-Akt (Fig. 3D). Therefore, we suggest it is likely that Akt activation is necessary for the SP-mediated migration of ST2 cells. Additionally, we demonstrated that the Src kinase inhibitor PP2 also blocked the migration of 

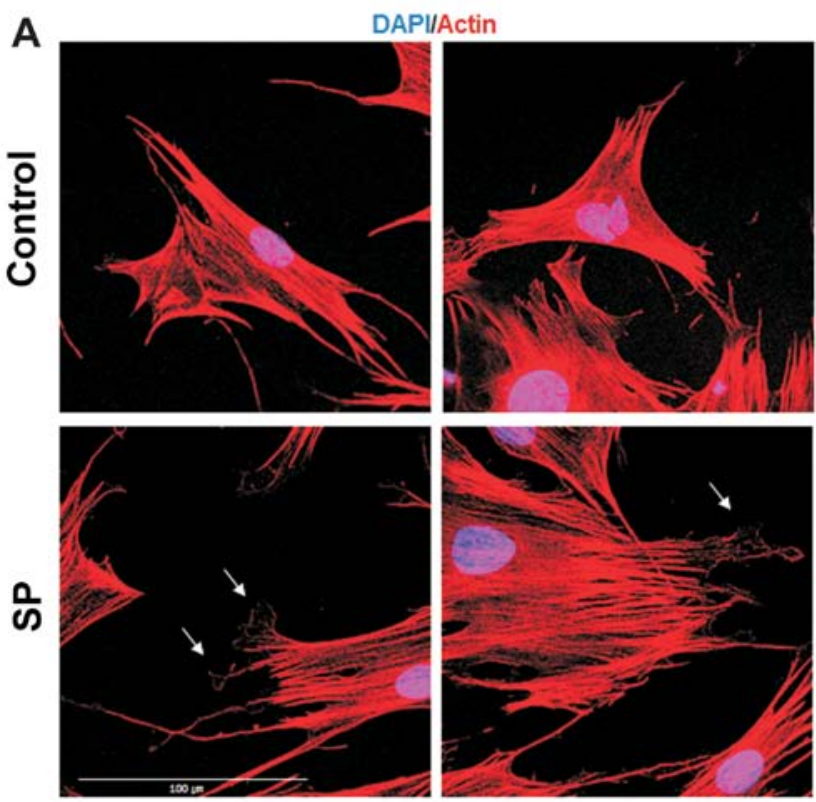

B

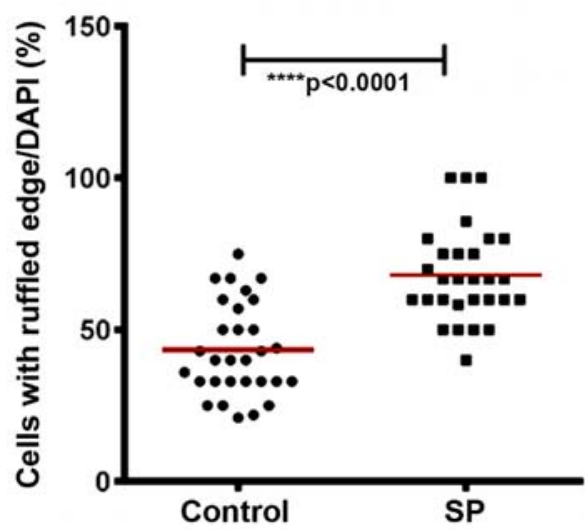

Figure 4. Substance P (SP) treatment increases the number of ST2 cells with membrane ruffles. (A) Immunocytochemical analysis of ST2 cells after 30 min of $300 \mathrm{nM}$ SP treatment using phalloidin staining to study changes in the actin cytoskeleton. (B) Quantitative analysis of ST2 cells presenting membrane ruffles after $30 \mathrm{~min}$ of $300 \mathrm{nM}$ SP treatment. Control cells were treated with the appropriate amount of SP solvent (5\% acetic acid). White arrows indicate membrane ruffles. Red lines indicate mean values; p-values were calculated using the Student's t-test. Scale bar, $100 \mu \mathrm{m}$.

ST2 cells in response to SP (Fig. 3C). This suggests that Src kinase plays an important role in the SP-mediated migration of ST2 cells. Importantly, as shown in Fig. 3E, we observed that LY294002 reduced the SP-induced increase in p-FAK, thus suggesting that Akt acts upstream of FAK to induce ST2 migration in response to SP.

SP treatment increases membrane ruffling on ST2 cells. Cell movement is driven by a continuous reorganization and turnover of the actin cytoskeleton that causes the formation of protrusive structures in the cell membrane at the leading edge such as filopodia, lamellipodia, ruffles and podosomes $(32,33)$. In order to evaluate whether SP induces changes in the actin cytoskeleton, we performed actin immunostaining of the ST2 cells after 30 min of SP treatment. We noted that SP increased the number of the ST2 cells that exhibited ruffled membrane
A
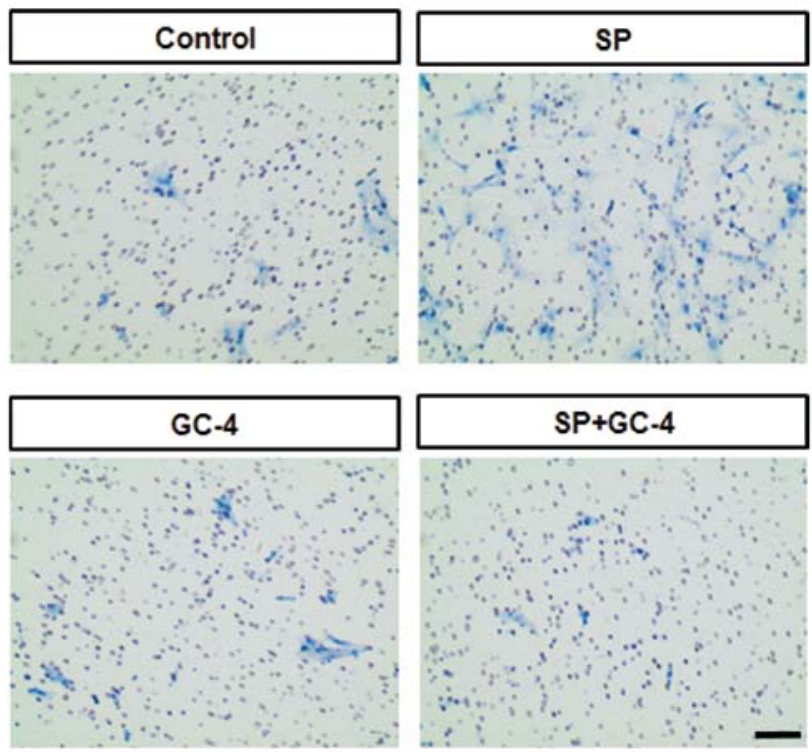

B

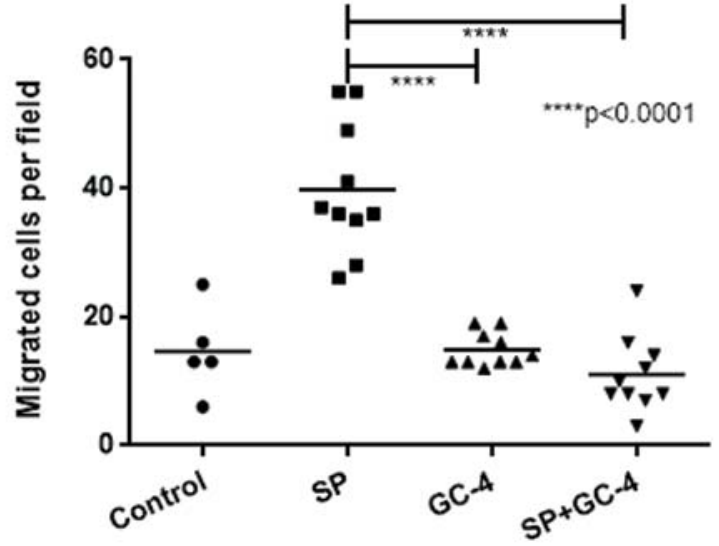

Figure 5. N-cadherin blocking antibody inhibits the migration of ST2 cells in response to substance $\mathrm{P}$ (SP). (A) Representative images of migrated ST2 cells following $300 \mathrm{nM}$ SP treatment and (B) the quantitative analysis of the migrated ST2 cells. ST2 cells were exposed for $30 \mathrm{~min}$ to $40 \mu \mathrm{g} / \mathrm{ml}$ $\mathrm{N}$-cadherin blocking antibody (GC-4). The control group was pre-treated with $40 \mu \mathrm{g} / \mathrm{ml}$ mouse IgG. Five fields of the lower chamber membrane were photographed and the migrated cells were then counted. Scale bar, $100 \mu \mathrm{m}$. Black lines indicate the mean values; p-values were calculated using the Student's t-test.

structures, compared with the control group (Fig. 4). These data suggest that SP modulates the actin dynamics to induce the migration of ST2 cells.

$N$-cadherin is required for SP-mediated migration of ST2 cells. Several studies have demonstrated that the cell adhesion molecule $\mathrm{N}$-cadherin is involved in the migration of various cell types under several developmental and stimulatory conditions (20-22). We sought to determine whether N-cadherin is necessary for the SP-mediated migration of ST2 cells. In ST2 cells exposed to the N-cadherin functional blocking antibody (GC-4), a decrease in SP-induced chemotactic migration was noted (Fig. 5), even though SP did not markedly affect the mRNA or protein levels of N-cadherin in the ST2 cells (data not shown). Thus, our data suggest that $\mathrm{N}$-cadherin is required for the migration of ST2 cells in response to SP. 


\section{Discussion}

To the best of our knowledge, the present study is the first to demonstrate that SP induces the migration of the BM-MSC-like cell line, ST2 cells, through $\mathrm{N}$-cadherin as well as by activating ERKs and Akt. Our results also suggest that FAK and Src kinase are involved in the migration of ST2 cells in response to SP. However, in the present study we did not show how SP activates these important signaling pathways and regulates $\mathrm{N}$-cadherin function in order to enhance the migration of ST2 cells in response to SP. Nonetheless, our findings suggest the cellular and molecular mechanisms responsible for the SP-mediated migration of BM-MSCs.

A previous study has shown that SP increases the activity of ERKs and the cellular proliferation of human BM-MSCs (9). However, that study did not demonstrate whether ERK activity is required for the increase in cellular proliferation which is mediated by SP treatment. Interestingly, even though in the present study we observed an increase in ERK after SP treatment, our previous study showed that SP does not induce the proliferation of ST2 cells (23). According to the present data, ERK activity seems to mediate the migration of ST2 cells in response to SP. Indeed, we noted that inhibiting ERK actvity blocked the SP-mediated migration of the ST2 cells to a similar extent as the antagonist of SP receptor (NK-1), RP 67580. On the contrary, we have previously reported that SP induces the proliferation of OP9 cells, another BM-derived MSC-like cell line, but ERK is not activated (23). Therefore, it is likely that the activation of ERKs in response to SP contributes to the cellular migration of BM-MSCs, not to their cellular proliferation.

Previous studies have shown that the phosphorylation of FAK at Tyr 397 induces cellular migration and that this phosphorylation is upregulated through PI3K/Akt $(34,35)$. The results of our present study showed that exposure of ST2 cells to the inhibitor of PI3K, LY294002, blocked the activation of Akt and the migration of the ST2 cells in response to SP. Importantly, LY294002 inhibited the phosphorylation of FAK induced by SP treatment. Therefore, we suggest that PI3K/Akt acts upstream of FAK, regulating the cellular migration of ST2 cells in response to SP.

Surprisingly, we found that N-cadherin mediated the cellular migration of the ST2 cells in response to SP. The impairment of $\mathrm{N}$-cadherin-mediated intercellular interactions by exposing the ST2 cells to $\mathrm{N}$-cadherin specific blocking antibody (clone GC-4), as previously described (36), inhibited SP-mediated migration of ST2 cells. However, we did not observe any changes in the mRNA and protein levels of N-cadherin following SP treatment (data not shown). We hypothesize that SP induces changes in the cellular localization of $\mathrm{N}$-cadherin and that these changes, rather than its expression level, are involved in the regulation of cellular migration by $\mathrm{N}$-cadherin. It has previously been demonstrated that neural crest cells undergo chemotactic migration towards SDF-1 via the polarized activity of Rac1 that is dependent on $\mathrm{N}$-cadherin-mediated intercellular interactions (22). It is likely that N-cadherin regulates Rac1 activity in ST2 cells in order to induce the chemotactic migration in response to SP. Further studies are thus necessary to elucidate how SP modulates $\mathrm{N}$-cadherin and how $\mathrm{N}$-cadherin promotes the migration of ST2 cells in response to SP.
This study revealed the molecular and cellular mechanisms that mediate the migration of the BM-derived MSC-like cell line ST2 in response to SP. Further research is thus warranted to confirm the involvement of such mechanisms in BM-MSC migration mediated by SP, and to examine their specific roles and interactions which enhance the migration of BM-MSCs.

\section{Acknowledgements}

This study was supported by the Korean Health Technology R\&DProject, Ministry of Health and Welfare, Republic of Korea (HI13C1479), the Basic Science Research Program through the National Research Foundation of Korea (NRF) funded by the Ministry of Education (NRF-2012R1A1A2042265), and the Bio and Medical Technology Development Program of the National Research Foundation (NRF) funded by the Ministry of Science, ICT and Future Planning (NRF-2012M3A9C6050485).

\section{References}

1. Ko IK, Lee SJ, Atala A and Yoo JJ: In situ tissue regeneration through host stem cell recruitment. Exp Mol Med 45: e57, 2013.

2. Rennert RC, Sorkin M, Garg RK and Gurtner GC: Stem cell recruitment after injury: lessons for regenerative medicine. Regen Med 7: 833-850, 2012.

3. Williams AR and Hare JM: Mesenchymal stem cells: biology, pathophysiology, translational findings, and therapeutic implications for cardiac disease. Circ Res 109: 923-940, 2011.

4. Lau TT and Wang DA: Stromal cell-derived factor-1 (SDF-1): homing factor for engineered regenerative medicine. Expert Opin Biol Ther 11: 189-197, 2011.

5. Wan M, Li C, Zhen G, Jiao K, He W, Jia X, Wang W, Shi C, Xing Q, Chen YF, et al: Injury-activated transforming growth factor $\beta$ controls mobilization of mesenchymal stem cells for tissue remodeling. Stem Cells 30: 2498-2511, 2012.

6. Zhang F, Tsai S, Kato K, Yamanouchi D, Wang C, Rafii S, Liu B and Kent KC: Transforming growth factor-beta promotes recruitment of bone marrow cells and bone marrow-derived mesenchymal stem cells through stimulation of MCP-1 production in vascular smooth muscle cells. J Biol Chem 284: 17564-17574, 2009.

7. Veevers-Lowe J, Ball SG, Shuttleworth A and Kielty CM: Mesenchymal stem cell migration is regulated by fibronectin through $\alpha 5 \beta 1$-integrin-mediated activation of PDGFR- $\beta$ and potentiation of growth factor signals. J Cell Sci 124: 1288-1300, 2011.

8. Hong HS, Kim Y, Yoon KJ and Son Y: A new paradigm for stem cell therapy: substance-P as a stem cell-stimulating agent. Arch Pharm Res 34: 2003-2006, 2011.

9. Hong HS, Lee J, Lee E, Kwon YS, Lee E, Ahn W, Jiang MH, Kim JC and Son Y: A new role of substance P as an injuryinducible messenger for mobilization of CD29(+) stromal-like cells. Nat Med 15: 425-435, 2009.

10. Bergfeld SA and DeClerck YA: Bone marrow-derived mesenchymal stem cells and the tumor microenvironment. Cancer Metastasis Rev 29: 249-261, 2010.

11. Mishra PJ, Mishra PJ, Glod JW and Banerjee D: Mesenchymal stem cells: flip side of the coin. Cancer Res 69: 1255-1258, 2009.

12. Gharibi B, Ghuman MS and Hughes FJ: Akt- and Erk-mediated regulation of proliferation and differentiation during PDGFR $\beta$-induced MSC self-renewal. J Cell Mol Med 16: 2789-2801, 2012.

13. Rodrigues M, Griffith LG and Wells A: Growth factor regulation of proliferation and survival of multipotential stromal cells. Stem Cell Res Ther 1: 32, 2010

14. Bulj Z, Duchi S, Bevilacqua A, Gherardi A, Dozza B, Piccinini F, Adalgisa Mariani G, Lucarelli E, Giannini S, Donati D and Marmiroli S: Protein kinase B/AKT isoform 2 drives migration of human mesenchymal stem cells. Int J Oncol 42: 118-126, 2013

15. Gao H, Priebe W, Glod J and Banerjee D: Activation of signal transducers and activators of transcription 3 and focal adhesion kinase by stromal cell-derived factor 1 is required for migration of human mesenchymal stem cells in response to tumor cellconditioned medium. Stem Cells 27: 857-865, 2009. 
16. Huttenlocher A and Horwitz AR: Integrins in cell migration. Cold Spring Harb Perspect Biol 3: a005074, 2011.

17. Sieg DJ, Hauck CR, Ilic D, Klingbeil CK, Schaefer E, Damsky CH and Schlaepfer DD: FAK integrates growth-factor and integrin signals to promote cell migration. Nat Cell Biol 2: 249-256, 2000

18. Sieg DJ, Hauck CR and Schlaepfer DD: Required role of focal adhesion kinase (FAK) for integrin-stimulated cell migration. J Cell Sci 112: 2677-2691, 1999.

19. Gumbiner BM: Regulation of cadherin-mediated adhesion in morphogenesis. Nat Rev Mol Cell Biol 6: 622-634, 2005.

20. Suyama K, Shapiro I, Guttman M and Hazan RB: A signaling pathway leading to metastasis is controlled by $\mathrm{N}$-cadherin and the FGF receptor. Cancer Cell 2: 301-314, 2002.

21. Park KS, Dubon MJ and Gumbiner BM: N-cadherin mediates the migration of MCF-10A cells undergoing bone morphogenetic protein 4-mediated epithelial mesenchymal transition. Tumour Biol 36: 3549-3556, 2015.

22. Theveneau E, Marchant L, Kuriyama S, Gull M, Moepps B, Parsons $\mathrm{M}$ and Mayor R: Collective chemotaxis requires contactdependent cell polarity. Dev Cell 19: 39-53, 2010.

23. Dubon MJ and Park KS: Substance P enhances the proliferation and migration potential of murine bone marrow-derived mesenchymal stem cell-like cell lines. Exp Ther Med 9: 1185-1191, 2015.

24. Li L and Jiang J: Regulatory factors of mesenchymal stem cell migration into injured tissues and their signal transduction mechanisms. Front Med 5: 33-39, 2011.

25. Alsayed Y, Ngo H, Runnels J, Leleu X, Singha UK, Pitsillides CM, Spencer JA, Kimlinger T, Ghobrial JM, Jia X, et al: Mechanisms of regulation of CXCR4/SDF-1 (CXCL12)-dependent migration and homing in multiple myeloma. Blood 109: 2708-2717, 2007.

26. Colston JT, de la Rosa SD and Freeman GL: Impact of brief oxidant stress on primary adult cardiac fibroblasts. Biochem Biophys Res Commun 316: 256-262, 2004.

27. Wang JF, Park IW and Groopman JE: Stromal cell-derived factor-1alpha stimulates tyrosine phosphorylation of multiple focal adhesion proteins and induces migration of hematopoietic progenitor cells: roles of phosphoinositide-3 kinase and protein kinase C. Blood 95: 2505-2513, 2000.
28. Zha YH, He JF, Mei YW, Yin T and Mao L: Zinc-finger transcription factor snail accelerates survival, migration and expression of matrix metalloproteinase- 2 in human bone mesenchymal stem cells. Cell Biol Int 31: 1089-1096, 2007.

29. Schmidt A, Ladage D, Schinköthe T, Klausmann U, Ulrichs C, Klinz FJ, Brixius K, Arnhold S, Desai B, Mehlhorn U, et al: Basic fibroblast growth factor controls migration in human mesenchymal stem cells. Stem Cells 24: 1750-1758, 2006.

30. Zhao X and Guan JL: Focal adhesion kinase and its signaling pathways in cell migration and angiogenesis. Adv Drug Deliv Rev 63: 610-615, 2011

31. Fu X, Han B, Cai S, Lei Y, Sun T and Sheng Z: Migration of bone marrow-derived mesenchymal stem cells induced by tumor necrosis factor-alpha and its possible role in wound healing. Wound Repair Regen 17: 185-191, 2009.

32. Bailly $\mathrm{M}$ and Condeelis J: Cell motility: insights from the backstage. Nat Cell Biol 4: E292-E294, 2002.

33. Pollard TD and Borisy GG: Cellular motility driven by assembly and disassembly of actin filaments. Cell 112: 453-465, 2003.

34. Higuchi M, Kihara R, Okazaki T, Aoki I, Suetsugu S and Gotoh Y: Akt1 promotes focal adhesion disassembly and cell motility through phosphorylation of FAK in growth factor-stimulated cells. J Cell Sci 126: 745-755, 2013.

35. Turecková J, Vojtechová M, Krausová M, Sloncová E and Korínek V: Focal adhesion kinase functions as an Akt downstream target in migration of colorectal cancer cells. Transl Oncol 2: 281-290, 2009.

36. Li G, Satyamoorthy K and Herlyn M: N-cadherin-mediated intercellular interactions promote survival and migration of melanoma cells. Cancer Res 61: 3819-3825, 2001. 